Bull. Mater. Sci., Vol. 3, Number 4, December 1981, pp. 447-454. (C) Printed in India.

\title{
SEM-studies of tin-iodide whiskers
}

\author{
C C DESAI and J L RAI \\ Department of Physics, Sardar Patel University, Vallabh Vidyanagar 388 120, India
}

MS received 17 March 1981 ; revised 27 May 1981

\begin{abstract}
Optical and scanning electron microscopic observations have been made of tin-iodide whiskers crystals grown by the gel-method. Most of the whiskers have been found to be of two types: (i) hollow and (ii) solid whiskers. The observations indicate that the formation of hollow whiskers is possible with or without the presence of impurities. By using electron micro probe, the overgrowth of tin-tetra iodide crystals on tin-iodide whisker crystals have been identified. A growth mechanism for tin-iodide whiskers have been suggested. The implications are discussed.
\end{abstract}

Keywords. Gel method; whisker crystals; micro crystallites; SEM; electron microprobe

\section{Introduction}

Since tin-whiskers were discovered by Compton et al (1951) whiskers have attracted special attention of many investigators for their characteristic forms and physical properties such as mechanical strength. A wide variety of materials have been grown in whiskers forms from various media and methods. There have been many observations of whiskers growing from porous substances and bulk crystal (Amelinckx 1958; Arnold and Koonce 1953). Sears (1957) and Amelinckx (1959) proposed the growth mechanism for rod-like and hollow whiskers. Desai and Rai (1981) have reported the growth of tin-iodide whisker crystals by the gel-method. Tin-iodide is an important crystal for many organic preparations and is used as an accelerator for butyl rubber vulcanization (Khristov and Bonchev 1977). It has also high colour rendering property (Zollwag and Frost 1966) when used in an electric lamp. The present authors have made detailed observations of tin-iodide whisker crystals using optical microscope, scanning electron microscope (SEM) and electron microprobe analysis. The present paper describes the observations and a possible mechanism for the growth of tin-iodide whiskers.

\section{Experimental}

Tin-iodide whisker crystals have been grown by the gel technique under the controlled reaction between $\mathrm{SnCl}_{2}$ and $\mathrm{KI}$ in silica gel medium (Desai and Rai 1981). The crystallization of whiskers were usually completed in 4-6 days. The whisker crystals have been identified by $\mathrm{x}$-ray analysis.

For SEM studies, the samples were prepared by first mounting the whisker crystal 
on a $15 \mathrm{~mm}$ diameter aluminium stud. The samples were then coated with Au-Pd film in a Carl Zeiss (78785 DDR) vacuum evaporator at a vacuum of $10^{-5}$ torr, to a thickness of $100 \AA$. The coating ensures electrical conductivity of the sample. The samples were analysed with a Cambridge SEM-model S4-10 scanning electron microscope, equipped with a storage oscilloscope and a secondary electron detector. A $25 \mathrm{keV}$ electron beam was used. The concentration of tin and iodine was studied by scanning the electron beam over the sample and monitoring the resultant Sn and I radiations. The pulses from the detected $x$-rays were imaged on the storage oscilloscope until the location of high concentration areas became apparent. To get detailed informations about the chemical analysis, the whiskers were examined at different locations.

\section{Results and discassion}

The tin-iodide $\left(\mathrm{SnI}_{2}\right)$ whiskers are orange to yellowish in colour with smooth and well developed $\{010\}$ habit faces. The average length of whisker crystals is found to be about $10 \mathrm{~mm}$ and thickness $30-250$ microns. They have been usually found in the form of straight needles, ribbons or thin platelets and ribbon whiskers grown over the other ribbon whiskers.

Figure 1 shows a typical SEM-micrograph of the longitudinal section of whisker crystals. It has been clearly seen that there are two types of whiskers: (i) solid whiskers (marked as A) and (ii) hollow whiskers (marked as B). Figures $2 a$ and $2 b$ represent SEM-micrographs at higher magnifications showing the transverse sections of the hollow whiskers. This configuration may be thought to result from the growth of hollow columns, similar to the case of CdS whiskers (Wood, 1959). The x-ray rotation photograph (figure 3) taken along the whisker axis gives a $\langle 010\rangle$ orientation for the whiskers. Striations parallel to $\langle 010\rangle$ directions were usually observed on the outer surfaces of most of the whisker crystals, and are illustrated in figure 4 . These striations indicate the possibility of the prism faces being obtained by the growth of whisker clusters growing parallel to the $b$-axis, and a process of space filling between the whiskers. Such an array of whiskers is also observed in calcium sulphide crystals (Dierssen and Gloor 1972) and ZnS (Iuanaga and Shibata 1972). The opening in the prism faces may be explained on the basis of a possible difference in the velocity of growth of whiskers. Figure 5 illustrates another common feature observed in the present work, the branching and tapering of whiskers. The photograph indicates that even though the tip of a growing whisker accidentally met another whisker, the growth continued further.

A large number of microcrystallites found to be over growing on some whiskers is illustrated in figure 6 . In order to investigate the chemical composition of these overgrown microcrystallites a beam of electron was focussed on them for electron microprobe analysis. Figure 7 shows the average energy spectrum of overgrown crystals while figure 8 is that of $\mathrm{SnI}_{2}$ whisker crystals. It is readily evident from these figures that the peaks correspond to the energies of characteristic $x$-rays of tin and iodine for $\mathrm{SnI}_{4}$ and $\mathrm{SnI}_{2}$ crystals respectively. Apart from tin and iodine, few traces of calcium and chlorine were also identified (as impurities) in $\mathrm{SnI}_{2}$ and $\mathrm{SnI}_{4}$ crystals. On the basis of the results of Paconci (1970) it may be assumed that these impurities are present in sufficient amounts to promote the growth of hollow whiskers. 

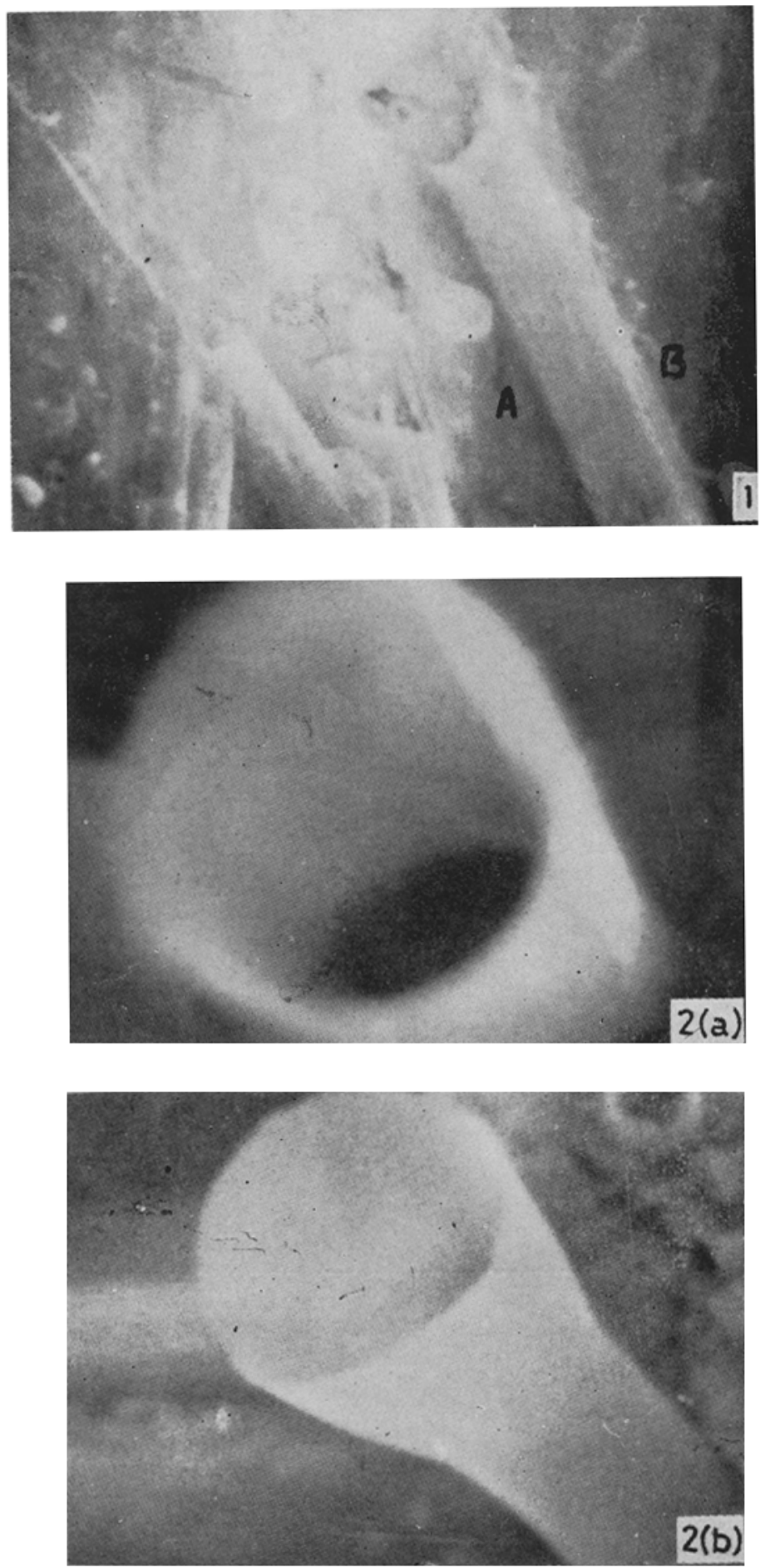

Figure 1. Projecting end views of solid and hollow whisker crystals. ( $\times 1250)$. Figure 2 a. $(\times 10,000)$ and $2 b(\times 5,000)$. The typical hollow whisker crystals viewed from the top. 


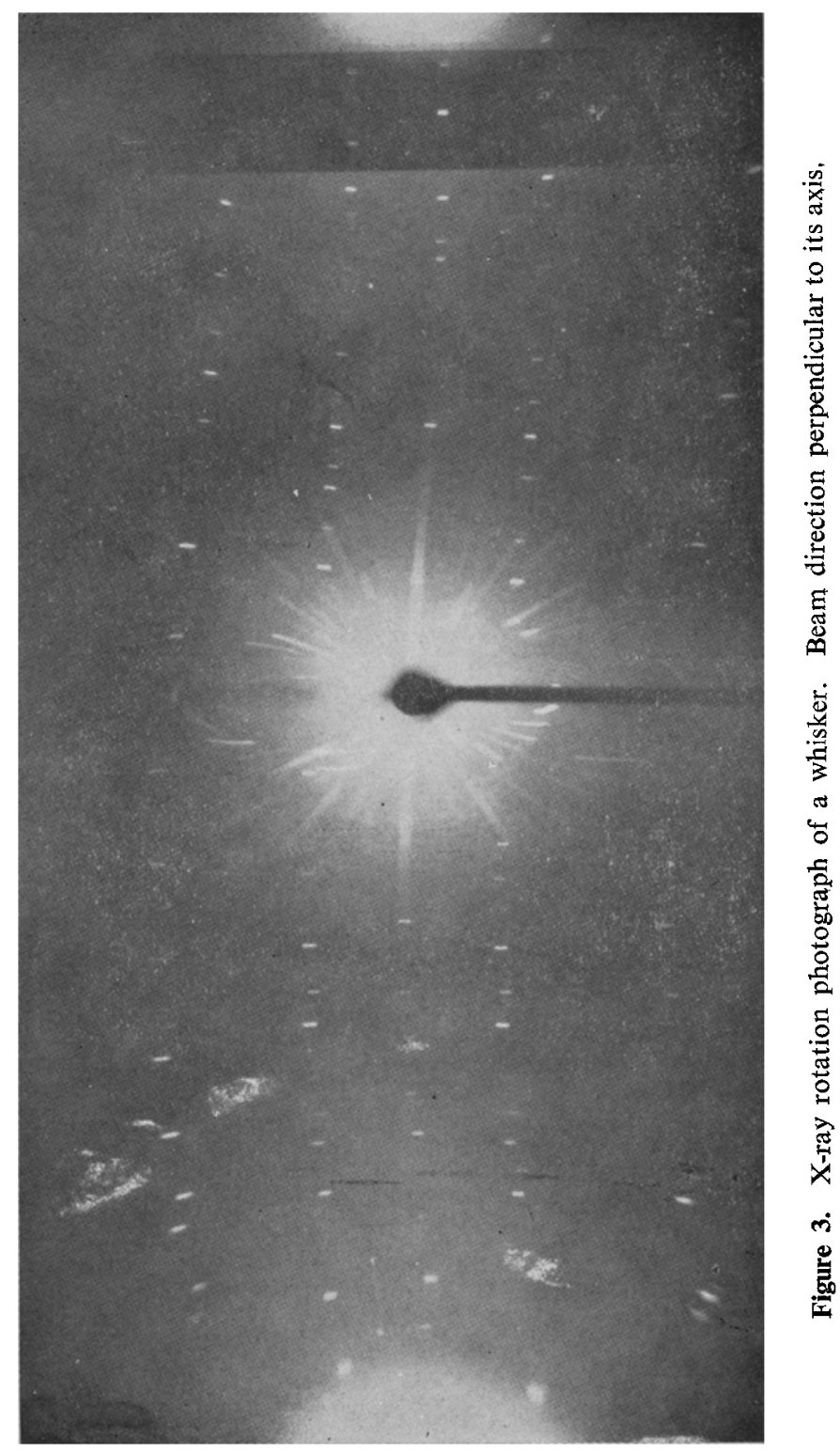




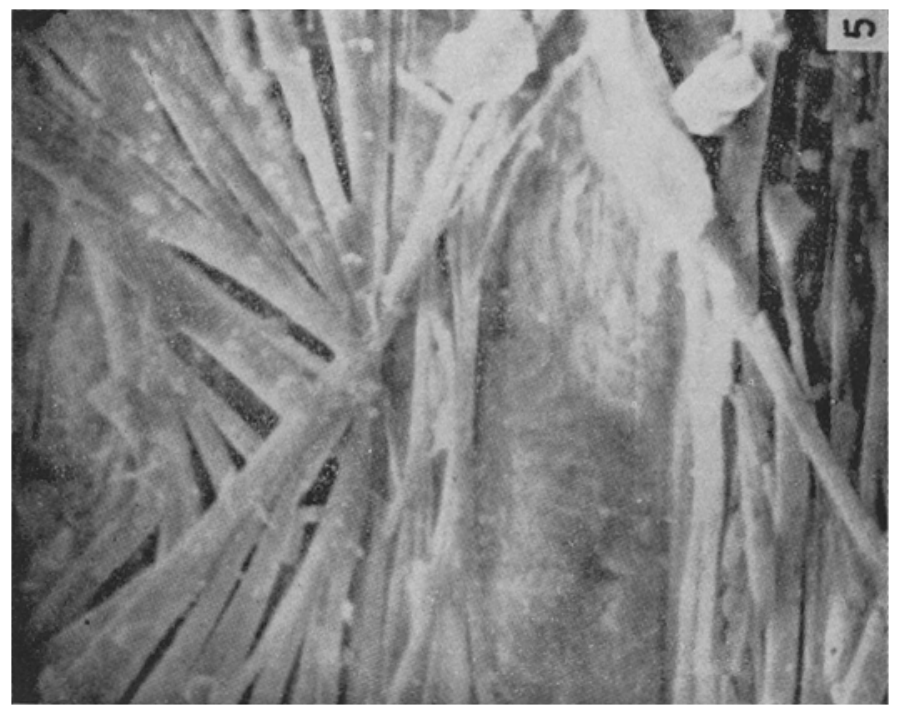

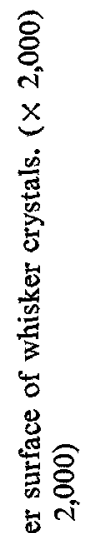

离

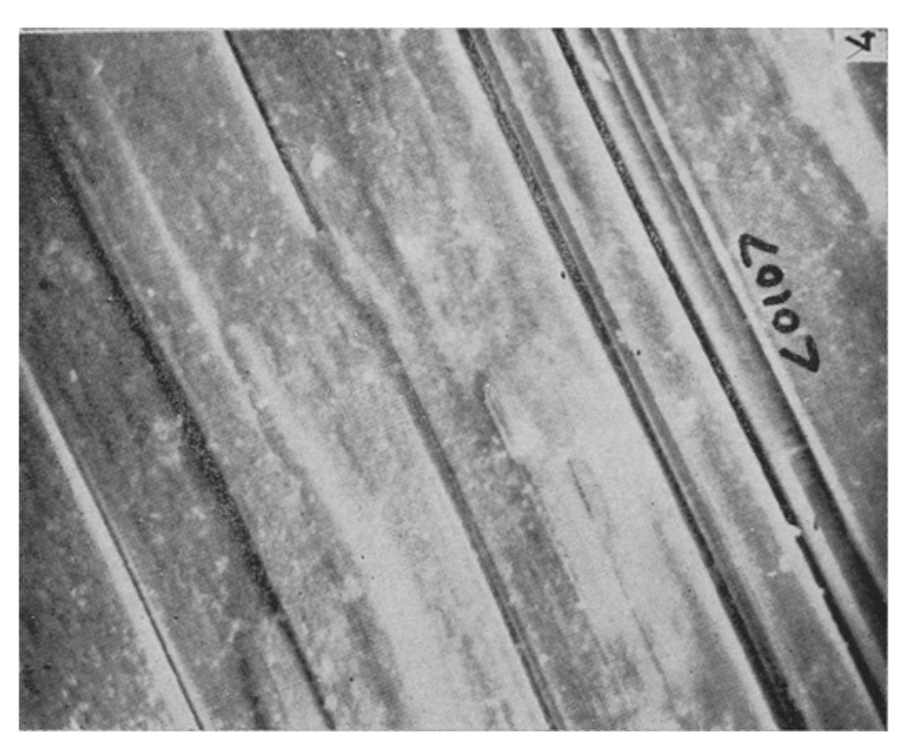

홍

응

윰굴

矛

悹

on

起䓵

$\dot{r}$ in

总密 


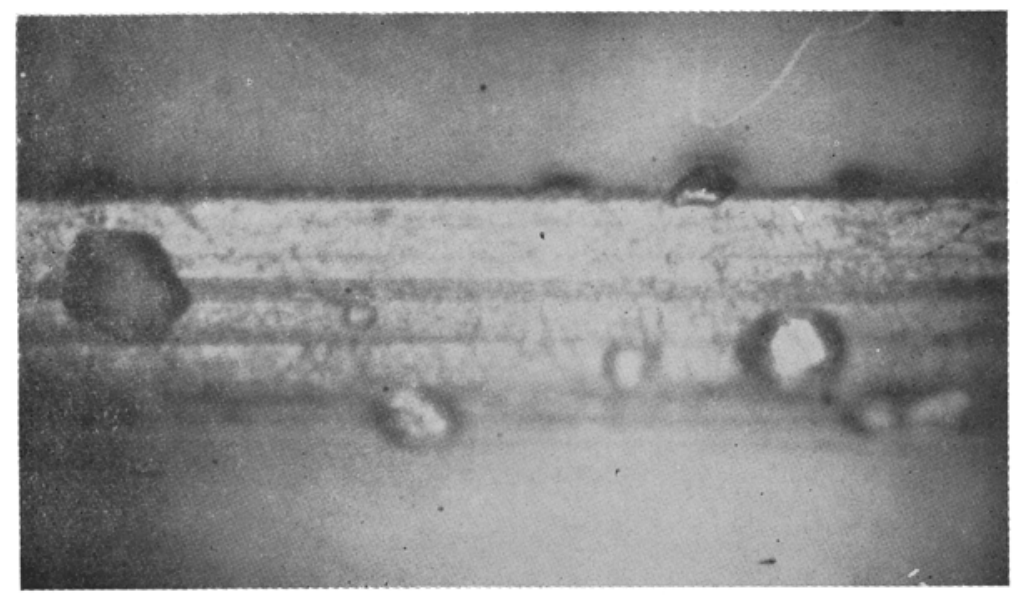

Figure 6. Overgrowth of microcrystallites on surfaces of $\mathrm{SnI}_{2}$ whiskers. $(\times 80)$. 


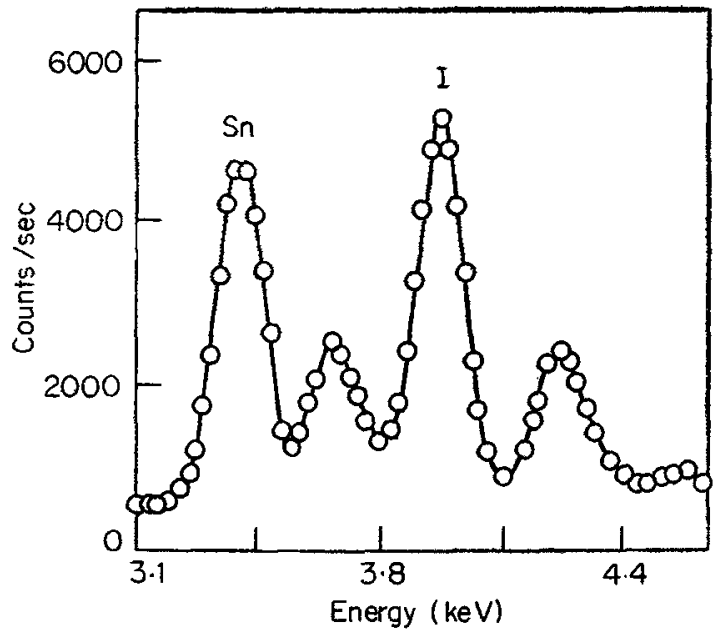

Figure 7. Average energy curves of $\mathrm{SnI}_{4}$ crystals.

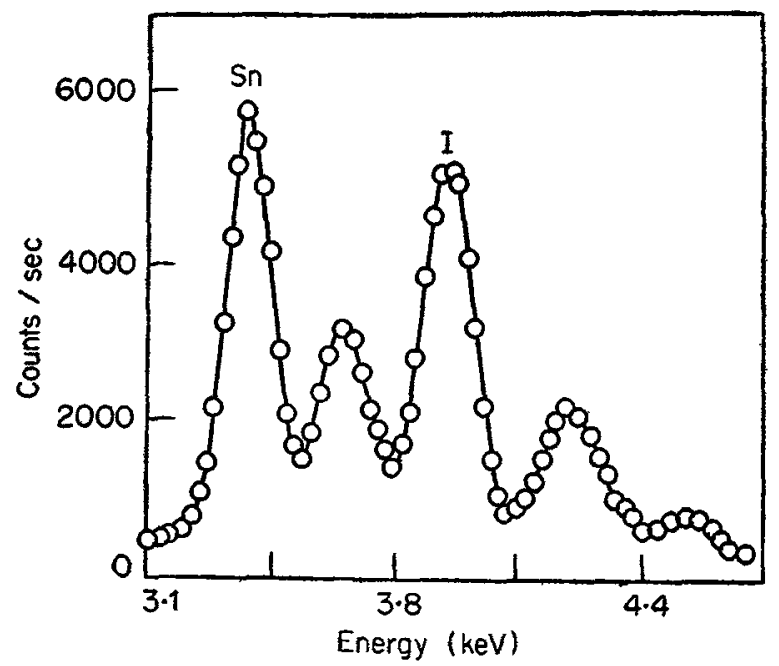

Figure 8. Average energy curves of $\mathrm{SnI}_{2}$ crystals.

The morphological study of whiskers makes it possible to distinguish the growth of whiskers by stages, as in growth of inorganic salts in supersaturated water solution (Charuslay and Rush 1958). The first stage can be characterized by a rapid linear growth and during the second stage broadening of whisker takes place through layer growth of surrounding faces. The separate layers originate usually at the base and grow in a direction to the tip, forming a fine striation on the walls of whiskers. The observed striated structure may be thought to represent the thickening stage of whisker growth. The following conclusions may be drawn from this study:

(i) It is possible to grow solid and hollow whiskers of $\mathrm{SnI}_{2}$ by using the gel-method

(ii) The experimental observations in the present work suggest that the formation of hollow whiskers is possible in the presence of impurities. 
(iii) By using electron microprobe analysis, the overgrown microcrystallites have been identified as $\mathrm{SnI}_{4}$ crystals grown on $\mathrm{SnI}_{2}$ whiskers.

\section{Acknowledgements}

The authors thank Professor A R Patel and Professor M S Joshi for their keen interest in the present work. One of us (JLR) thanks UGC, New Delhi for the award of a Teacher Fellowship, during the tenure of which the present work was carried out. One of us (CCD) is grateful to Mrs G C Desai for her good wishes for the work.

\section{References}

Amelinckx S 1958 Physica 24390

Amelinckx S 1959 J. Chem. Phys. 311687

Arnold S M and Koonce S 1953 J. Appl. Phys. 24365

Charuslay P and Rush E 1958 Philos. Mag. 3508

Compton K G, Mendizza A and Arnold S M 1951 Corrosion 7327

Desai C C and Rai J L $1981 J$. Crystal Growth (in press)

Dierssen G H and Galoor T 1972 J. Crystal Growth 1699

Iuanaga $H$ and Shibata N 1972 Jpn. J. Appl. Phys. 1121

Khristov D and Bonchev T 1977 Kautch, Gummi, Kunstst 30845

Paonci C 1970 J. Mater. Sci. 5918

Sears G W 1957 J. Chem. Phys. 261549

Thomas E and Miltoo M 1972 (Shell oil co.) US 3668109 (Cl 208-10 C 10 g) 06 January.

Wood J 1959 Br. J. Appl. Phys. 10529

Zollwag R J and Frost L S 1966 Bull. Am. Phys. Soc. 11745 